International Journal of Thermofluid Science and Technology (2020)

Volume 7, Issue 1, Paper No. 20070101

https://doi.org/10.36963/IJTST.20070101

IITST

\title{
Heat Transfer Analysis on Peristaltic Transport of a Jeffery Fluid in an Inclined Elastic Tube with Porous Walls
}

\author{
G.Manjunatha' $^{1}$, C. Rajashekhar ${ }^{2}$, Hanumesh Vaidya ${ }^{3 *}$, K. V. Prasad ${ }^{3}$, Saraswati ${ }^{3}$, B. B. Divya1 \\ 1 Department of Mathematics, Manipal Institute of Technology, Manipal Academy of Higher Education, \\ Manipal-576104, Karnataka, India \\ 2 Bhaskaracharya Study Chair, Karnataka State Akkamahadevi Women's University, Vijayapura-586108, \\ Karnataka India \\ 3 Department of Mathematics, Vijayanagara Sri Krishnadevaraya University, Jnana Sagara Campus, Vinayaka \\ Nagar, Ballari-583 105, Karnataka, India
}

Received: 30 July 2019; Received in revised form: 10 December 2019; Accepted: 20 December 2019; Published online10 January 2020

(C) Published at www.ijtf.org

\begin{abstract}
This article analyses the effects of heat transfer and thermal conductivity on the peristaltic transport of Jeffery fluid through an inclined elastic tube with porous walls. The velocity slip and convective boundary conditions are taken into account. The modeled governing equations are solved analytically by considering the long wavelength and small Reynolds number approximations. The closed-form solutions are obtained for velocity, flow rate, and the theoretical determination of flow rate is calculated with the help of equilibrium condition given by Rubinow and Keller. A parametric analysis has been presented to study the effects of Jeffery parameter, thermal conductivity, Darcy number, the angle of inclination, velocity slip, Biot number, amplitude ratio, Prandtl number, and Eckert number on velocity, flow rate, and temperature are scrutinized. The streamlines show that the bolus moves with the same speed as that of the wave and further the study reveals that an increase in the Biot number reduces the magnitude of the temperature.
\end{abstract}

Keywords: Angle of inclination; Convective conditions; Jeffery parameter; Variable thermal conductivity; Velocity slip

\section{Introduction}

The investigation of heat transfer effects along with slip conditions on peristalsis has bought the attention of researchers in past decades due to their extensive application in sanitary fluid transport, heart-lung machines, laser therapy, hypothermia treatment, transport of corrosive fluids and coldness cryosurgery (Jaggy et al., 2000). The examination of heat transfer is concerned with temperature and the flow of heat. Where temperature decides the amount of thermal energy

*Corresponding e-mail: hanumeshvaidya@gmail.com 
G. Manjunatha et al.

International Journal of Thermofluid Science and Technology (2020), Volome 7, Issue 1, Paper No. 20070101

available while heat flow indicates the movement of thermal energy from one place to another. In general, the heat transfer can happen in three unique classes: conduction, convection, and radiation. Among the three classifications, the convective method of heat transfer assumes an indispensable part in numerous applications. Mainly, it is utilized as a part of oxygenation and hemodialysis. Keeping this in mind, numerous scientists have explored the fluid characteristics by taking convective boundary conditions alongside variable thermal conductivity (Hayat et al., 2013; Alsaedi et al., 2013; Abbasi et al., 2015; Hayat et al., 2016; Sayed et al., 2016; Abdul, 2017; Srinivasa, 2017; Prasad et al., 2018(a)). The initial studies on the peristaltic transport of a Newtonian/nonNewtonian fluid has been carried out by many researchers by taking different assumptions and configuration (Latham, 1966; Burns and Parkes, 1967; Raju and Devanathan, 1972; Vajravelu et al.,(2016)(a); Vajravelu et al., (2016)(b); Prasad et al., 2017; Manjunatha and Rajashekhar, 2018; Prasad et al., 2018(b); Vaidya et al., 2019 (a-d)). Among the several non-Newtonian models, the Jeffery model is more significant in describing the flow of blood in arteries. The studies on the use of the Jeffery model was carried out by Hayat et al. (2007) to investigate the peristaltic transport in a circular tube. Nadeem and Akram (2010) analyzed the peristaltic transport in a rectangular duct and obtained the exact solutions for pressure rise and pressure gradient. Further, several authors used the Jeffery model for investigating the peristaltic transport with different geometries and assumptions to represent the specific living situation (Vajravelu et al., 2011; Bhatti and Abbas, 2016; Selvi et al., 2017).

The flow of biological fluids through porous media plays a vital role in understanding the various mechanism in gallbladder, lungs, blood vessel movement, etc. These applications of porous media in biofluid dynamics and biomechanics have attracted researchers in recent times. In the human body, a large part of the tissues, like, muscles, ligaments, and bones form a deformable porous media. In such conditions, the presence of slip on the boundary because of the permeability of the walls has a necessary effect in reviewing the biological fluids. Thus, slip effects are more verbalized for fluids going through geometries which have flexible property, like blood vessels. This slip flow of fluids is used in the polishing of the internal cavities and artificial heart valves. The exploratory examinations on non-Newtonian fluids revealed the centrality of slip at the walls. The peristaltic flow of blood through a tube can be idealized better by considering slip and permeability. Studies on the utilization of porous walls on peristaltic transport have been initially explored by Elshehawey et al. (1999). Later, various researchers examined the impact of slip velocity on the peristaltic mechanism by using different models under different assumptions and geometries (Nadeem and Akram, 2011; Tripathi and Beg, 2012; El-Koumy et al., 2012; Manjunatha et al., $2019(a-c))$.

It is important to note that, the Poiseuille's law shows that for a fluid which is incompressible, the flux in the tube is a linear function of the pressure difference between the ends of the rigid tube through which it flows. Hence, the non-Newtonian fluids obey Poiseuille's law in most of the theoretical as well as experimental studies. The nonlinearity in vascular beds of warm-blooded creatures is ascribed to the flexible idea of veins and their immense distensibility. This elastic property of veins was first perceived by Young (1968). Further, Rubinow and Keller (1972) showed that the scope of the tube could be controlled by the strain in the dividers and the transmural weight contrast by accepting that the Poiseuille law holds locally. Consequently, there is a necessity for the subjective speculation of blood flow through elastic tubes. The stream designs bought by the 
G. Manjunatha et al.

International Journal of Thermofluid Science and Technology (2020), Volome 7, Issue 1, Paper No. 20070101

models with a rigid tube can't clarify the flow of blood in narrow arteries completely. Henceforth, it becomes important to consider the elasticity in the present model.

To the best of the authors' knowledge, no attempts have been made in the literature to investigate the role of variable thermal conductivity, convective boundary conditions and inclination on peristaltic transport of Jeffery fluid in an axisymmetric elastic tube with porous walls. The present investigation helps to fill the gap in this direction. The resulting modeled equations are solved analytically under the proper slip boundary conditions. The influence of pertinent parameters on velocity, flow rate, temperature, and streamlines are presented graphically. The outcomes of the present model help in understanding the complex physiological behavior of blood in the circumstances mentioned above, which intern helps medical people to investigate the blood flow in arteries a much better way than the earlier and, helps in modeling the heart-lung and dialysis machines.

\section{Mathematical Formulation}

The flow is considered to be laminar, steady, incompressible, fully-developed, axisymmetric and exhibiting peristalsis in an elastic tube with porous walls (See Fig. 1). The fluid is characterized by the Jeffery model and facilitates the choice of the cylindrical coordinate system to study the problem. The wall deformation due to the propagation of an infinite sinusoidal wave train of peristaltic waves is represented by

$$
h^{\prime}(z, t)=a+b \sin \left[\frac{2 \pi}{\lambda}(z-t)\right] .
$$

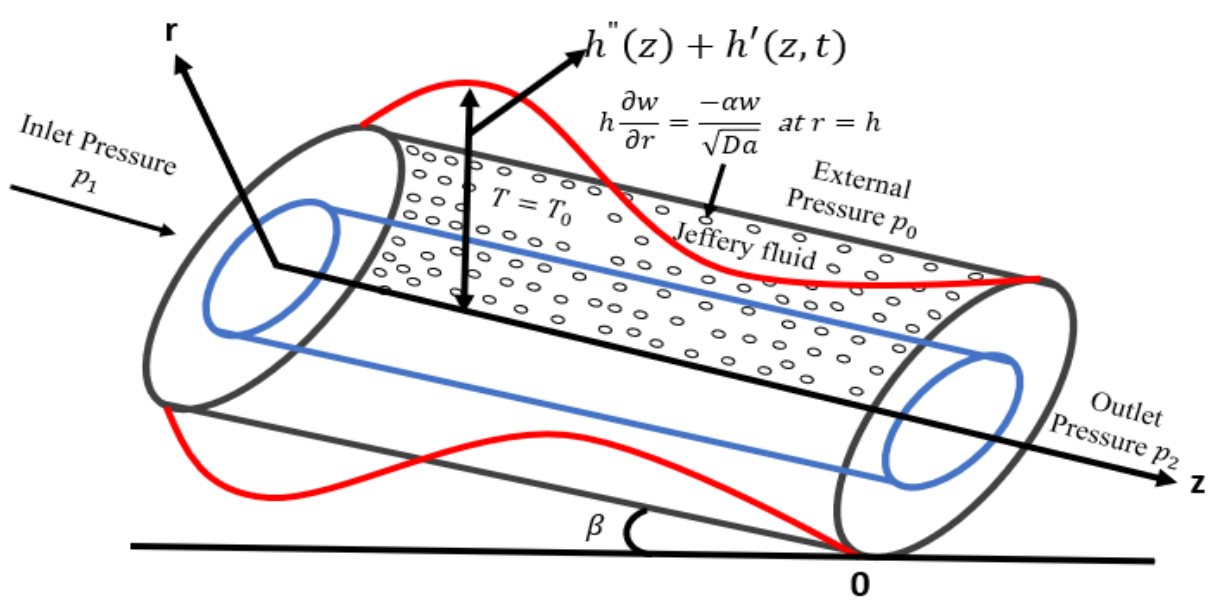

Fig. 1. Geometrical representation of Peristaltic waves in an elastic tube.

Consider the constitutive equations on the peristaltic transport of incompressible viscous Jeffery fluid represented by the following expressions

$\bar{T}=p \bar{I}+\bar{S}$, 


$$
\bar{S}=\frac{\mu}{1+\lambda_{1}}\left(\dot{\gamma}+\lambda_{2} \ddot{\gamma}\right)
$$

Where $\bar{T}$ is the Cauchy's stress tensor, $\bar{S}$ is the extra tensor, $\bar{I}$ is the identity tensor, $\lambda_{1}$ is the ratio of relaxation to retardation time, $\lambda_{2}$ is the retardation time and $\gamma$ is the shear rate and dots over the quantities indicate differentiation with respect to time.

The flow becomes steady in the wave frame $(r, \theta, z)$ moving with velocity $c$ away from the fixed frame $(R, \Theta, Z)$ given by

$$
r=R, z=Z-c t, \psi=\Psi-\frac{R^{2}}{2}, p(Z, t)=P(z),
$$

where $p$ and $P$ are pressures, $\psi$ and $\Psi$ are stream functions, in the wave and fixed frames of references, respectively.

The pressure $p$ remains constant at any axial station of the tube under the assumption of long wavelength approximation. Using the following nondimensional variables

$$
\begin{aligned}
& \bar{r}=\frac{r}{h^{\prime}}, \bar{z}=\frac{z}{\lambda}, \bar{t}=\frac{c t}{\lambda}, \overline{\tau_{r z}}=\frac{\tau_{r z}}{\mu\left(\frac{c}{h^{\prime}}\right)}, \bar{p}=\frac{p h^{\prime}}{\lambda c \mu}, \varepsilon=\frac{b}{a}, \operatorname{Pr}=\frac{\mu c_{p}}{k}, \bar{u}=\frac{u}{c}, \bar{w}=\frac{w}{c}, \\
& \theta=\frac{T-T_{0}}{T_{0}}, E c=\frac{c^{2}}{c_{p} T_{0}}, \delta=\frac{a}{\lambda}, \operatorname{Re}=\frac{\rho c a}{\mu}, F_{1}=\frac{\mu c}{\rho g a^{2}}, N=E c \times \operatorname{Pr} .
\end{aligned}
$$

The non-dimensional equations of motion and energy in the wave frame of reference, moving with speed $c$, under the lubrication approach is as follows:

$$
\begin{gathered}
\operatorname{Re} \delta\left(u \frac{\partial}{\partial r}+w \frac{\partial}{\partial z}\right) w=-\frac{\partial p}{\partial z}+\frac{1}{r} \frac{\partial}{\partial r}\left(r \tau_{r z}\right)+\delta \frac{\partial}{\partial r}\left(\tau_{z z}\right), \\
\operatorname{Re} \delta^{3}\left(u \frac{\partial}{\partial r}+w \frac{\partial}{\partial z}\right) u=-\frac{\partial p}{\partial r}+\frac{\delta}{r} \frac{\partial}{\partial r}\left(r \tau_{r r}\right)+\delta^{2} \frac{\partial}{\partial r}\left(\tau_{r z}\right), \\
\operatorname{Re} \delta \operatorname{Pr}\left(u \frac{\partial}{\partial r}+w \frac{\partial}{\partial z}\right) \theta=E c \operatorname{Pr}\left(\delta \frac{\partial u}{\partial r} \tau_{r r}+\frac{\partial w}{\partial r} \tau_{r z}+\delta^{2} \frac{\partial u}{\partial r} \tau_{z r}+\tau_{z z} \frac{\partial w}{\partial r} \delta\right) \\
+\frac{\partial^{2} \theta}{\partial r^{2}}+\frac{1}{r} \frac{\partial \theta}{\partial r}+\delta^{2} \frac{\partial^{2} \theta}{\partial z^{2}} .
\end{gathered}
$$

Where $u$ and $w$ are the radial and axial velocities, Re is the Reynolds number, $\theta$ is temperature, $\delta$ is wave number, $\operatorname{Pr}$ is the Prandtl number, $E c$ is the Eckert number, $r$ is radial coordinate, $\tau_{r r}$ is shear stress in radial coordinates $\tau_{z r}$ is shear stress in axial and radial coordinates, $\tau_{z z}$ is shear stress in axial coordinate and $\tau_{r z}$ is the shear stress along radial and axial coordinates.

Under the assumption of long wavelength and small Reynolds number, Eqs. (5) - (7) takes the form

$$
\frac{1}{r} \frac{\partial}{\partial r}(r \tau)=-\frac{\partial p}{\partial z}+\frac{\sin \beta}{F_{1}}
$$




$$
\begin{gathered}
0=\frac{\partial p}{\partial r}, \\
\frac{1}{r} \frac{\partial}{\partial r}\left(k(\theta) r \frac{\partial \theta}{\partial r}\right)=E c \operatorname{Pr}\left(-\frac{\partial w}{\partial r} \tau\right) .
\end{gathered}
$$

The corresponding non-dimensional boundary conditions are (Saffman, 1971; Abbasi et al., 2015)

$$
\begin{aligned}
& \quad h \frac{\partial w}{\partial r}=\frac{-\alpha w}{\sqrt{D a}}, \quad(1+\phi) \frac{\partial \theta}{\partial r}+B i \theta=0 \text { at } r=h, \\
& \frac{\partial \theta}{\partial r}=0, \tau_{r z} \text { is finite at } r=0 .
\end{aligned}
$$

Where $D a$ is the porous parameter (Darcy number), $\alpha$ is the velocity slip parameter, $\phi$ is the thermal conductivity, $k(\theta)=1+\phi \theta, \phi<<1, B i=\frac{l a}{k_{0}}$ is the Biot number and $l$ is the heat transfer coefficient of the wall and $\theta$ is the temperature.

The closed-form solutions are obtained for the velocity expression (8) and (9) satisfying the boundary conditions (11) and (12), we obtain the velocity as

$$
w=\frac{\left(1+\lambda_{1}\right)(P+f)}{4}\left[h^{2}-r^{2}+\frac{2 h^{2} \sqrt{D a}}{\alpha}\right] .
$$

Where $P=-\frac{\partial p}{\partial z}$ and $f=\frac{\sin \beta}{F_{1}}$ Using Eq. (10) together with the boundary conditions (11) and (12), we obtain an expression for temperature as

$$
\theta=\frac{N(P+f)^{2}\left(1+\lambda_{1}\right)}{64}\left[\frac{r^{4}}{4}-h^{3}\left\{\frac{h}{4}+\frac{1+\phi}{B i}\right\}\right] .
$$

2.1 Theoretical Determination of flux: Application to Flow Through an Artery

A theoretical calculation of the flux $Q$ is carried out for an incompressible Jeffery fluid through an elastic tube of radius $h(z, t)=h^{\prime}(z, t)+h^{\prime \prime}(z)$. The fluid is assumed to enter the tube with pressure $p_{1}$ and leave the tube with pressure $p_{2}$, while the pressure outside the tube is $p_{0}$. If $z$ denotes the distance along the tube from the inlet end, then the pressure $p(z)$ in the fluid at $z$ diminishes from $p(0)=p_{1}$ to $p(\lambda)=p_{2}$. The tube may contract or expand due to the difference in the pressure of the fluid $p(z)-p_{0}$. Subsequently, the cross-section of the tube may have a deformation due to the elastic property of the walls. Thus, the difference in pressure influences the conductivity $\sigma_{1}$ of the tube at $z$. We consider the conductivity $\sigma_{1}=\sigma_{1}\left[p(z)-p_{0}\right]$ to be a known function of the pressure difference $\left(p(z)-p_{0}\right)$. This conductivity is assumed to be the same as that of a uniform tube having an identical cross-section at $z$. The relation between $Q$ and the pressure gradient is given by

$$
Q=\sigma_{1}\left(p-p_{0}\right)(P+f) \text {. }
$$

Under the considerations of peristaltic motion and the elastic property of the tube wall, Eq. (15) can be written as, 


$$
\sigma_{1}\left(p-p_{0}\right)=\frac{F}{8}\left(h^{\prime}+h^{\prime \prime}\right)^{4}
$$

where $F=\left(1+\lambda_{1}\right)\left[1+\frac{4 \sqrt{D a}}{\alpha}\right]$ and $h^{\prime \prime}$ is the change in radius of the tube due to elasticity and is a function of pressure $p-p_{0}$ at each cross-section due to the Poiseuille flow. Equation (15) with the inlet condition $p(0)=p_{1}$ gives

$$
Q z=\int_{p(z)-p_{0}}^{p_{1}-p_{0}} \sigma_{1}\left(p^{\prime}\right) d p^{\prime}+\int_{0}^{1} \frac{F}{8} f\left(h^{\prime}+h^{\prime \prime}\right)^{4} d z
$$

where $p^{\prime}=p(z)-p_{0}$. Equation (17) gives $p(z)$ in terms of $z$ and $Q$ by setting $z=1$ and $p(1)=p_{2}$ we get $Q$ as,

Now, using Eq. (17) in Eq. (18), we have

$$
\begin{aligned}
& Q=\int_{p_{2}-p_{0}}^{p_{1}-p_{0}} \sigma_{1}\left(\mathrm{p}^{\prime}\right) \mathrm{dp}+\int_{0}^{1} \frac{F}{8} f\left(h^{\prime}+h^{\prime \prime}\right)^{4} \mathrm{dz} . \\
& Q=\frac{F}{8}\left[\int_{p_{2}-p_{0}}^{p_{1}-p_{0}}\left(h^{\prime}+h^{\prime \prime}\right)^{4} \mathrm{dp}^{\prime}+f\left(h^{\prime}+h^{\prime \prime}\right)^{4}\right] .
\end{aligned}
$$

Equation (19) can be solved if we explicitly know the function $h^{\prime \prime}\left(p-p_{0}\right)$. If $h^{\prime \prime}$ is known as a function of the tension $T\left(h^{\prime \prime}\right)$ or stress, then $h^{\prime \prime}\left(p^{\prime}\right)$ can be determined from the equilibrium condition (Rubinow and Keller, 1972) given by

$$
\frac{T\left(h^{\prime \prime}\right)}{h^{\prime \prime}}=p-p_{0}
$$

Rubinow and Keller (1972) carried out experimental investigations by controlling the static pressure-volume connection of a 4-cm long piece of a human iliac artery and gave an expression for tension in an elastic tube as:

$$
T\left(h^{\prime \prime}\right)=t_{1}\left(h^{\prime \prime}-1\right)+t_{2}\left(h^{\prime \prime}-1\right)^{5} .
$$

Using Eq. (21) with $t_{1}=13$ and $t_{2}=300$, Eq. (20) takes the following form

$$
\mathrm{dp}=\left[\frac{t_{1}}{h^{\prime \prime 2}}+t_{2}\left(4 h^{\prime \prime 3}-15 h^{\prime 2}+20 h^{\prime \prime}-10+\frac{1}{h^{\prime 2}}\right)\right] \mathrm{dh} " .
$$

Using Eq. (22), Eq. (19) can be written as

$$
Q=\frac{F}{8}\left[\int_{p_{2}-p_{0}}^{p_{1}-p_{0}}\left(h^{\prime}+h^{\prime \prime}\right)\left\{\frac{t_{1}}{h^{\prime 2}}+t_{2}\left(4 h^{\prime \prime 3}-15 h^{\prime \prime 2}+20 h^{\prime \prime}-10+\frac{1}{h^{\prime 2}}\right)\right\} d h^{\prime}+f\left(h^{\prime}+h^{\prime \prime}\right)^{4}\right] .
$$


G. Manjunatha et al.

International Journal of Thermofluid Science and Technology (2020), Volome 7, Issue 1, Paper No. 20070101

Letting $p=p_{1}$ and $p=p_{2}$ in Eq. (20) the solutions are obtained for $h_{1}^{\prime \prime}$ and $h_{2}^{\prime \prime}$ respectively.

Equation (23) can be rewritten as

$$
\begin{aligned}
Q= & \frac{F}{8}\left[\left(g\left(h_{1}^{\prime \prime}\right)-g\left(h_{2}^{\prime \prime}\right)\right)+f h_{2}^{\prime 4}\right] . \\
g(h) & =t_{1}\left(\frac{h^{\prime \prime 3}}{3}+2 h^{\prime} h^{\prime \prime}+6 h^{\prime 2} h^{\prime \prime}+4 h^{\prime 3} \log \log h^{\prime \prime}-\frac{h^{\prime 4}}{h^{\prime \prime}}\right)+t_{2}\left(\frac{h^{\prime \prime}}{2}+\frac{h^{\prime \prime 7}}{7}\left(16 h^{\prime}-15\right)\right. \\
& +\frac{h^{\prime \prime 2}}{2}\left(20 h^{\prime 4}-40 h^{\prime 3}+4 h^{\prime}\right)+\frac{h^{\prime \prime}}{5}\left(16 h^{\prime 3}-90 h^{\prime 2}+80 h^{\prime}-10\right)+\frac{h^{\prime \prime}}{6}\left(24 h^{\prime 2}-60 h^{\prime}+20\right) \\
& +\frac{h^{\prime \prime}}{3}\left(-15 h^{\prime 4}+80 h^{\prime 3}-60 h^{\prime 2}+1\right)+\frac{h^{\prime \prime}}{4}\left(4 h^{\prime 4}-60 h^{\prime 3}+120 h^{\prime 2}-40 h^{\prime}\right) \\
& \left.+h^{\prime \prime}\left(-10 h^{\prime 4}+6 h^{\prime 2}\right)+4 h^{\prime 3} \log \log h^{\prime \prime}-\frac{h^{\prime 4}}{h^{\prime \prime}}\right) .
\end{aligned}
$$

\section{Results and Discussion}

The present paper emphasizes on the combined effects of slip and heat transfer on the peristaltic transport of Jeffery fluid in an inclined elastic tube with porous walls. Figs. 2-9 are plotted to observe the effects of Jeffery parameter $\left(\lambda_{1}\right)$, the angle of inclination $(\beta)$, porous parameter $(D a)$, velocity slip parameter $(\alpha)$, amplitude ratio $(\varepsilon)$, elastic parameters $\left(t_{1}, t_{2}\right)$, inlet and outlet elastic radius $\left(h_{1}^{\prime \prime}, h_{2}^{\prime \prime}\right)$, Brinkmann number $(B r)$, thermal conductivity $(\phi)$ and Biot number $(B i)$ on velocity $(w)$, flow rate $(Q)$, streamlines $(\psi)$ and temperature $(\theta)$. MATLAB programming is used to plot these effects of physiological parameters.

Velocity profiles are plotted in Fig. 2 for different values $\lambda_{1}, D a, \varepsilon, \beta$ and $\alpha$. It is noticed that the velocity profiles are parabolic in nature with the maximum velocity at the center of the tube. From Figs. 2(a-d) it is observed that for a larger value of $\lambda_{1}, D a, \varepsilon$ and $\beta$ the magnitude of the velocity increases. Whereas, an increase in the value of $\alpha$ decreases the magnitude of the velocity. Figs. 3 and 4 are plotted to examine the effects of $\lambda_{1}, D a, \alpha, \beta, \varepsilon, t_{1}, t_{2}, h_{1}^{\prime \prime}$ and $h_{2}^{\prime \prime}$ on flow rate. Fig. 3(a) depicts the variation of $\lambda_{1}$ on flow rate. It is observed from the figure that an increase in the values of $\lambda_{1}$ enhances the flow rate in an elastic tube. Fig. 3(b) shows the variation of $D a$ on flow rate. It is noticed that an increase in the values of $D a$ increases the volume of flow rate. 

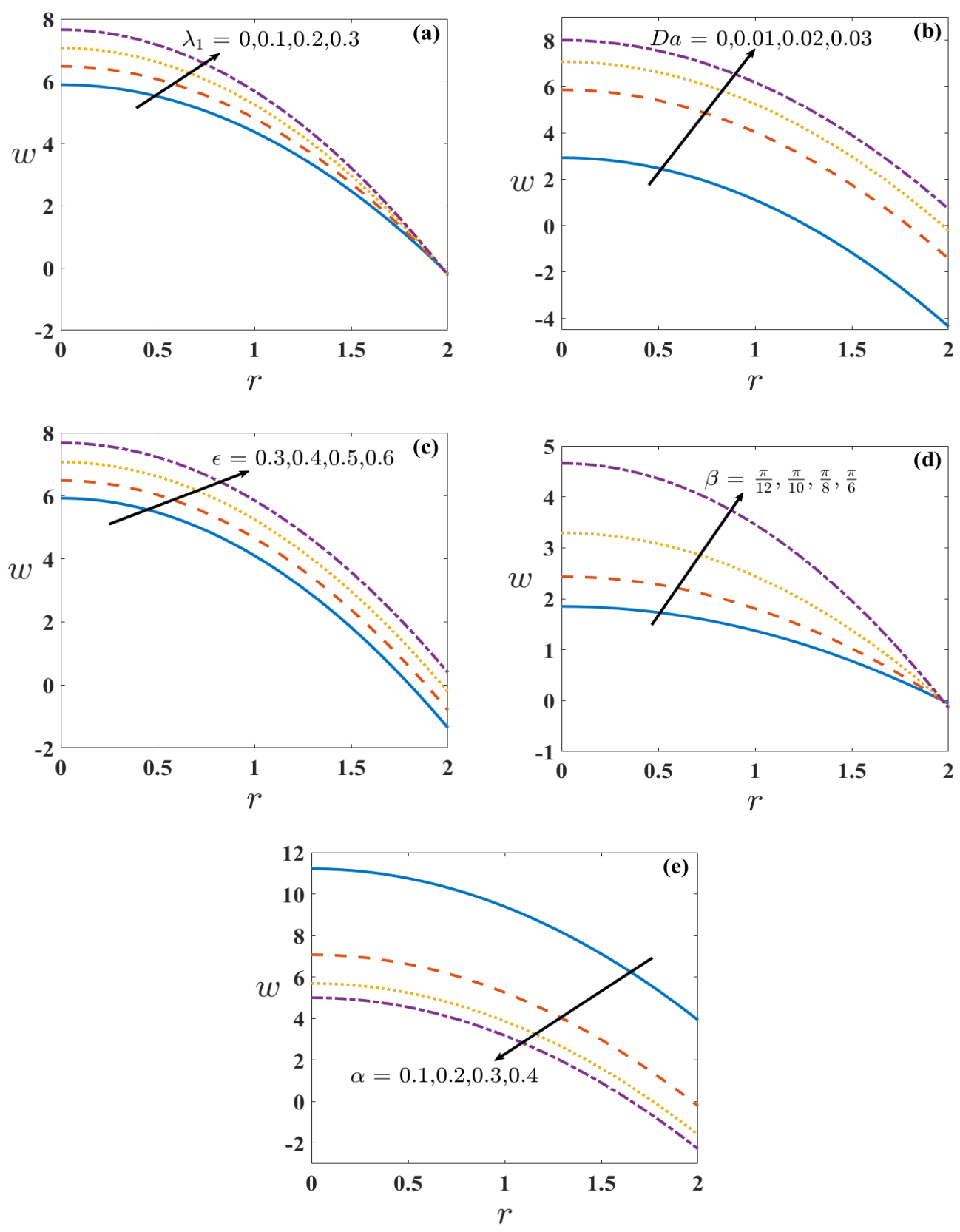

Fig. $2 w \mathbf{v} / \mathbf{s} r$ with varying (a) Jeffery parameter $\left(\lambda_{1}\right)$, (b) porous parameter $(D a)$, (c) amplitude ratio $(\varepsilon)$, (d) angle of inclination $(\beta)$ and (e) velocity slip parameter $(\alpha)$. 
G. Manjunatha et al.

International Journal of Thermofluid Science and Technology (2020), Volome 7, Issue 1, Paper No. 20070101
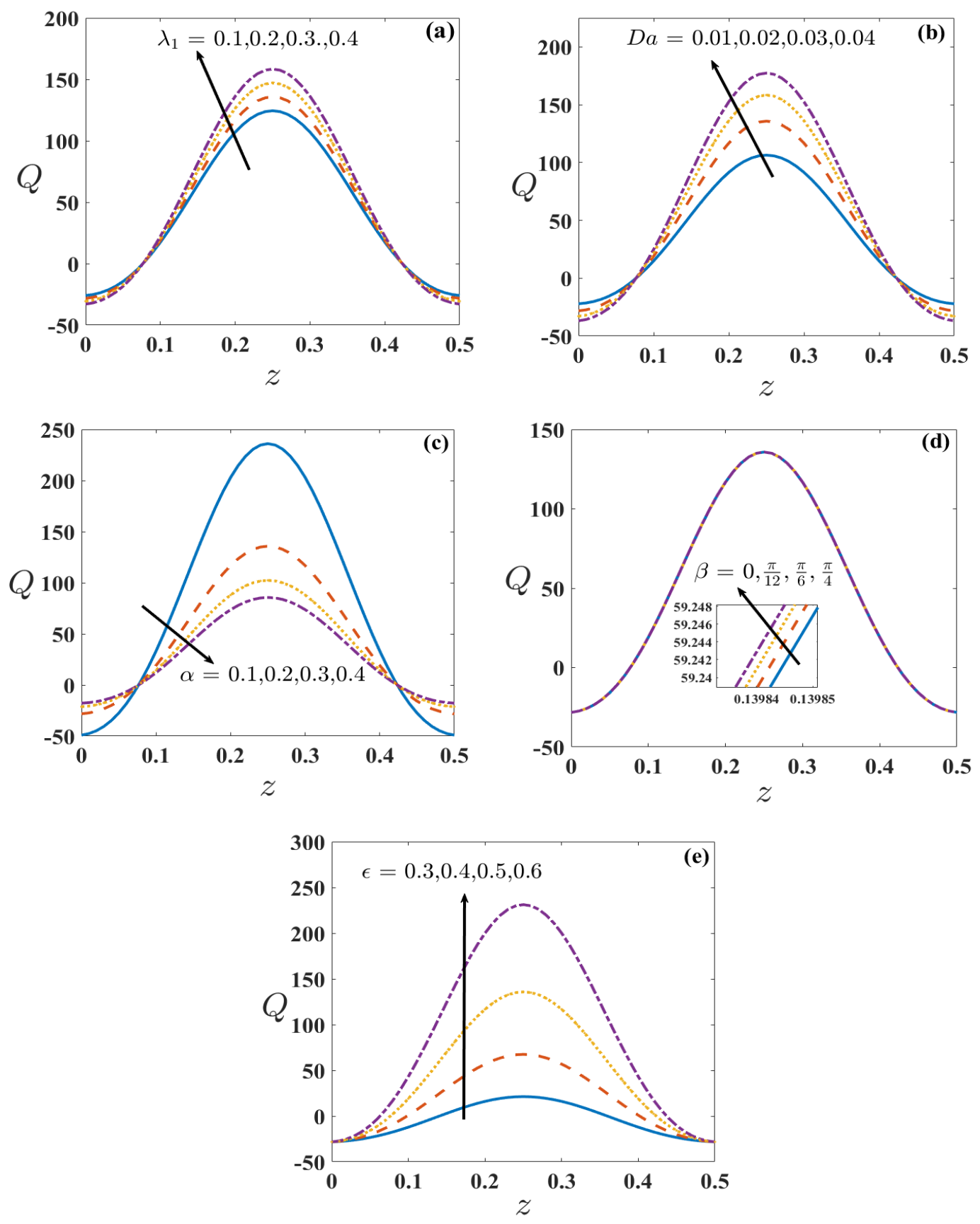

Fig. $3 Q$ v/s $z$ with varying (a) Jeffery parameter $\left(\lambda_{1}\right)$, (b) porous parameter $(D a)$, (c) velocity slip parameter $(\alpha),(\mathrm{d})$ angle of inclination $(\beta)$ and $(\mathrm{e})$ amplitude ratio $(\varepsilon)$. 
G. Manjunatha et al.

International Journal of Thermofluid Science and Technology (2020), Volome 7, Issue 1, Paper No. 20070101
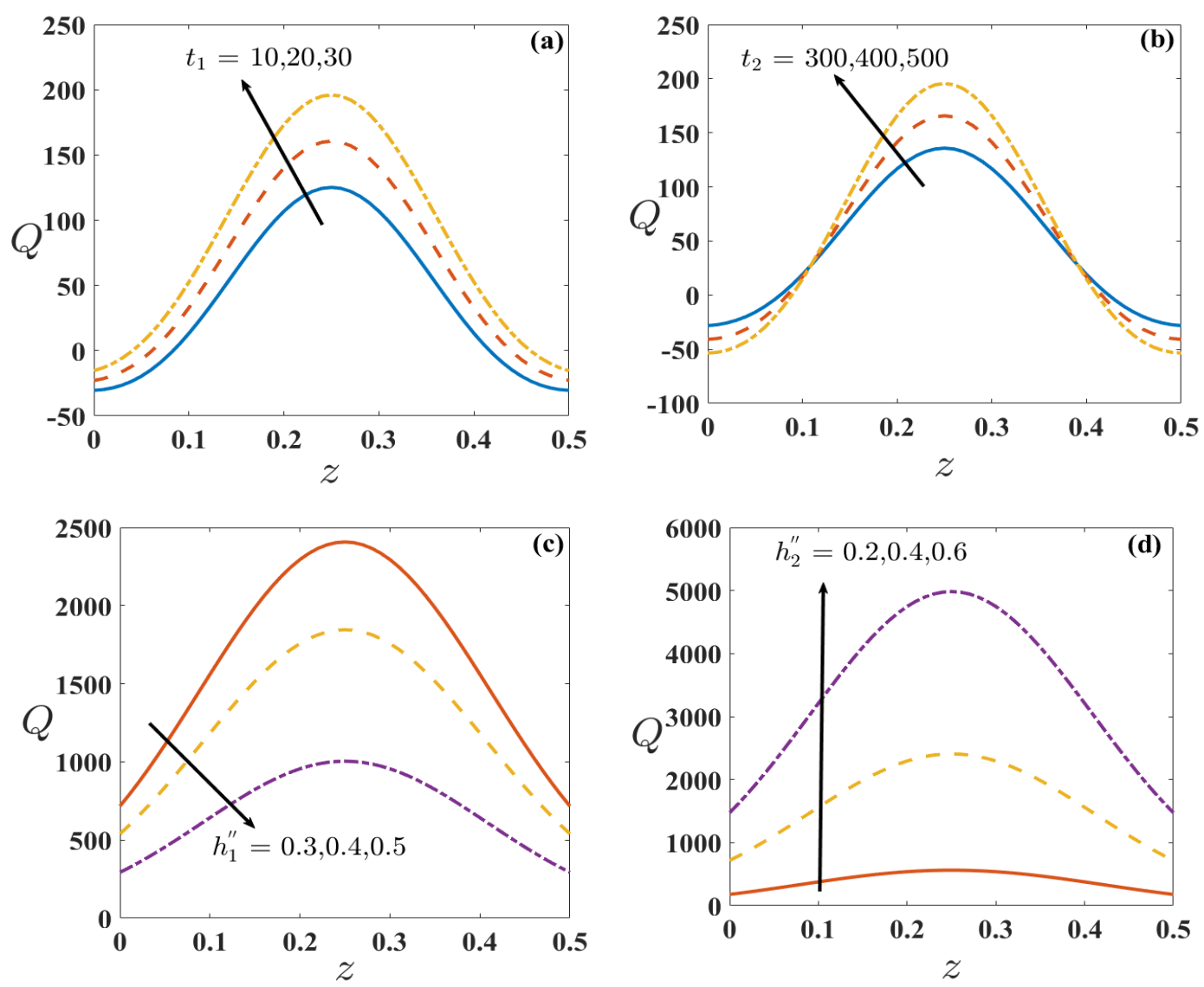

Fig. $4 Q \mathbf{v} / \mathbf{s} z$ with varying (a) elastic parameter $\left(t_{1}\right)$, (b) elastic parameter $\left(t_{2}\right)$, (c) inlet elastic radius $\left(h_{1}^{\prime \prime}\right)$ and (d) outlet elastic radius $\left(h_{2}^{\prime \prime}\right)$.

The influence of $\alpha$ on flow rate shows the opposite behavior as that of $D a$ (See Fig. 3(c)). Figure 3(d) portrays the variation of $\beta$ on flux. It is observed that the impact of increase in the angle $\beta$ increases the volume of flow rate. The variation of $\varepsilon$ on flow rate is illustrated in Fig. 3(e). It is clear from the figure that an increase in the value of $\varepsilon$ increases the volume of flow rate. Since $\varepsilon$ is the amplitude ratio, an increase in the value of $\varepsilon$ results in an increase in the wave height which in turn increases the flux.Figs. 4(a and b) are drawn to study the effects of $t_{1}$ and $t_{2}$ on $Q$ respectively. We see from these figures that an increase in the values of $t_{1}$ and $t_{2}$ enhances the flow rate. Further, the variation of inlet and outlet elastic radius $h_{1}^{\prime \prime}$ and $h_{2}^{\prime \prime}$ on $Q$ are plotted in Figs. 4(cand d). For a fixed value of $h_{2}^{\prime \prime}$, the effect of increasing values of $h_{1}^{\prime \prime}$ makes flow rate to decrease (See Fig. 4(c)). However, Fig.4(d) exhibits the opposite behavior when we fix $h_{1}^{\prime \prime}$ and vary $h_{2}^{\prime \prime}$. 
G. Manjunatha et al.

International Journal of Thermofluid Science and Technology (2020), Volome 7, Issue 1, Paper No. 20070101
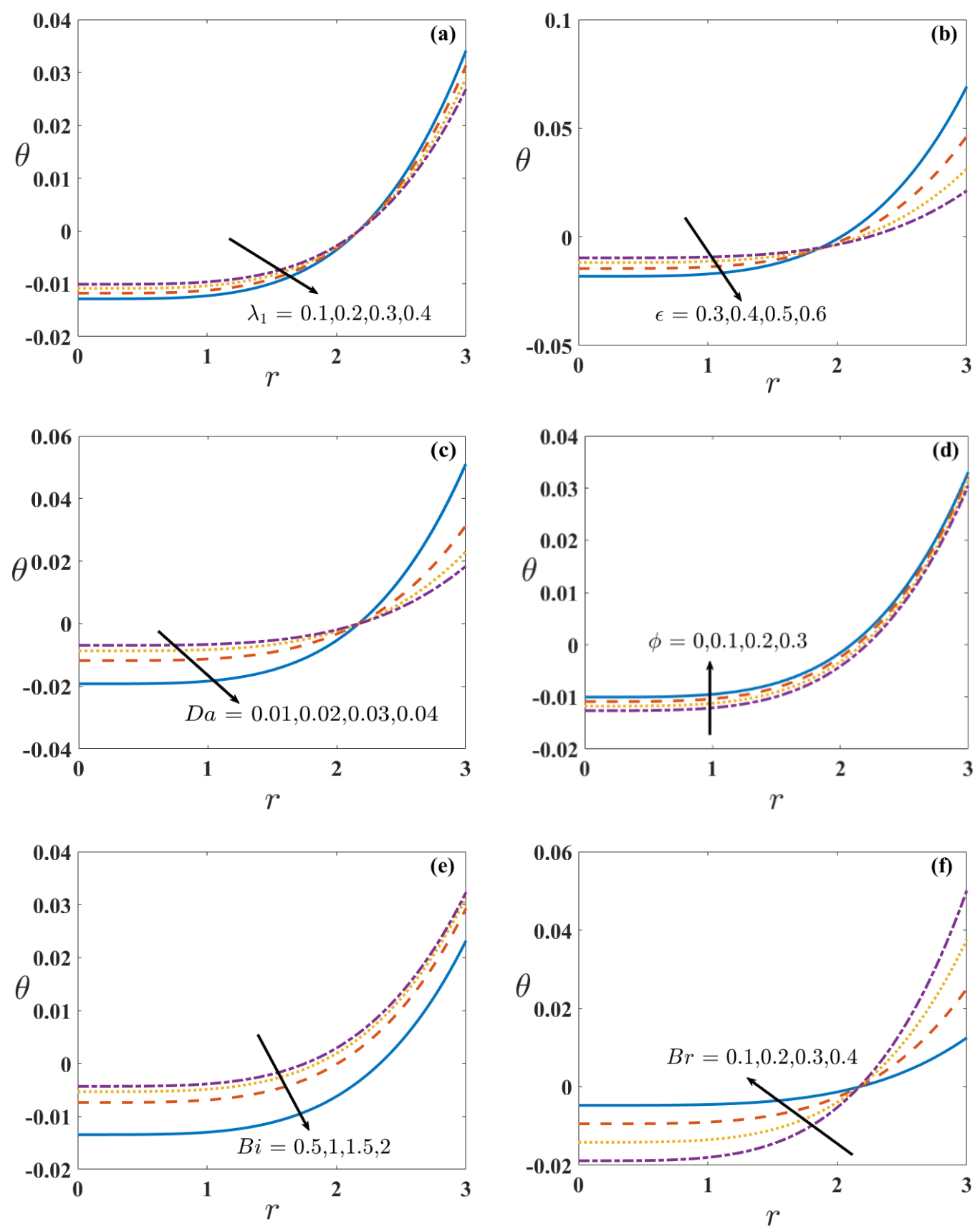

Fig. $5 \theta \mathrm{v} / \mathrm{s} r$ with varying(a) Jeffery parameter $\left(\lambda_{1}\right)$, (b) varyingamplitude ratio $(\varepsilon)$, (c) porous parameter $(D a)$, (d) thermal conductivity $(\phi)$, (e) Biot number $(B i)$ and (f) Brinkmann number $(\mathrm{Br})$. 
G. Manjunatha et al.

International Journal of Thermofluid Science and Technology (2020), Volome 7, Issue 1, Paper No. 20070101
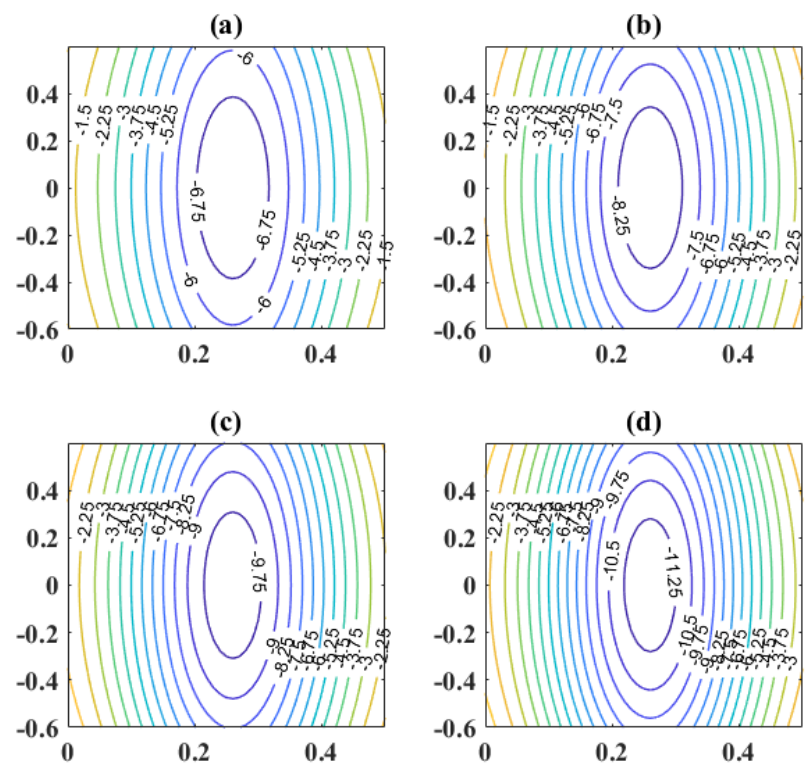

Figure 6. Streamlines for varying (a) $\lambda_{1}=0$, (b) $\lambda_{1}=0.2$, (c) $\lambda_{1}=0.4$ and (d) $\lambda_{1}=0.6$.
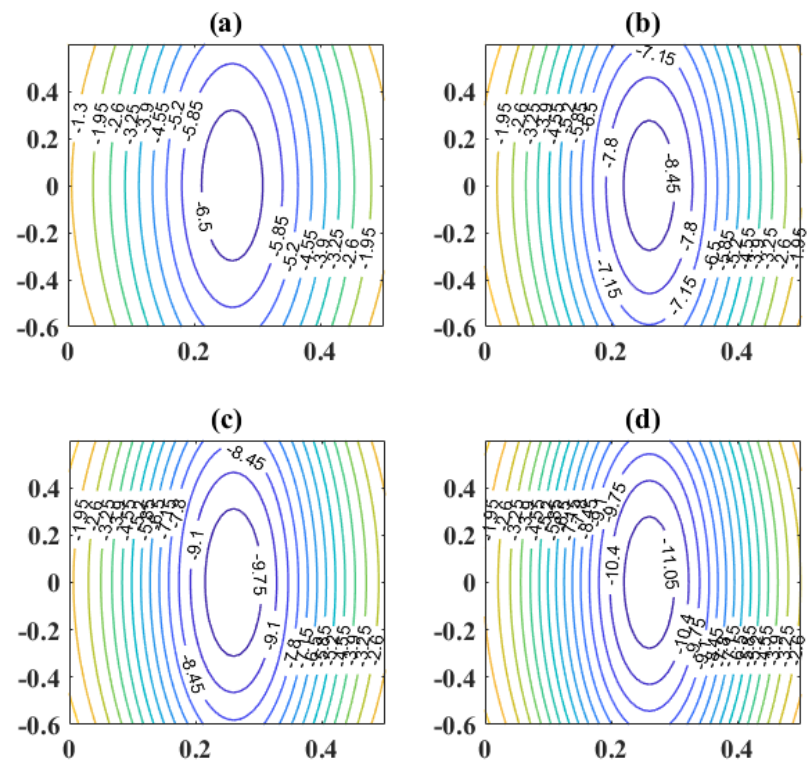

Figure 7. Streamlines for varying (a) $D a=0.01$, (b) $D a=0.02$, (c) $D a=0.03$ and (d) $D a=0.04$. 

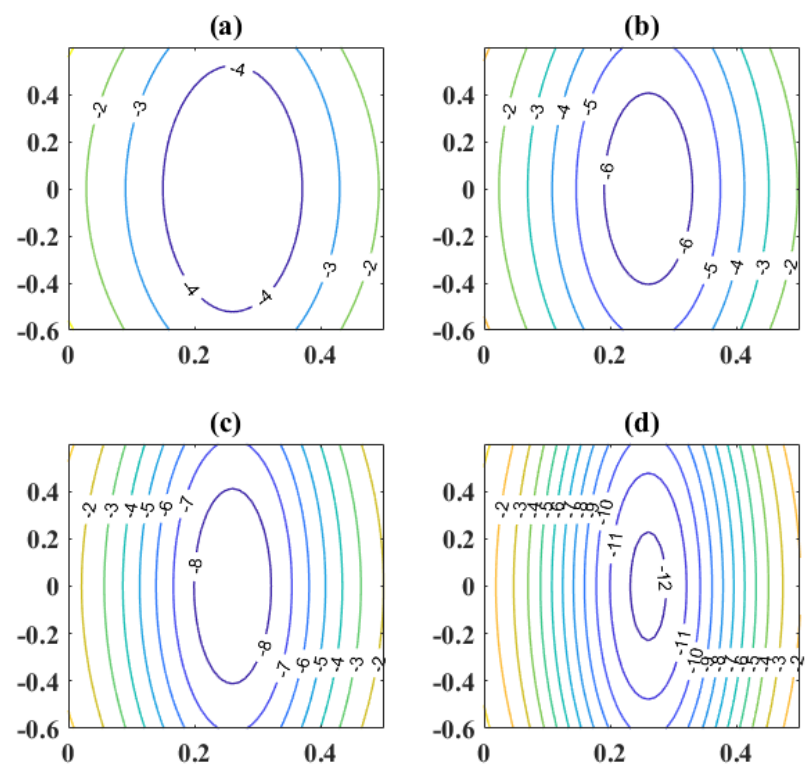

Figure 8. Streamlines for varying (a) $\varepsilon=0.3$, (b) $\varepsilon=0.4$, (c) $\varepsilon=0.5$ and (d) $\varepsilon=0.6$.
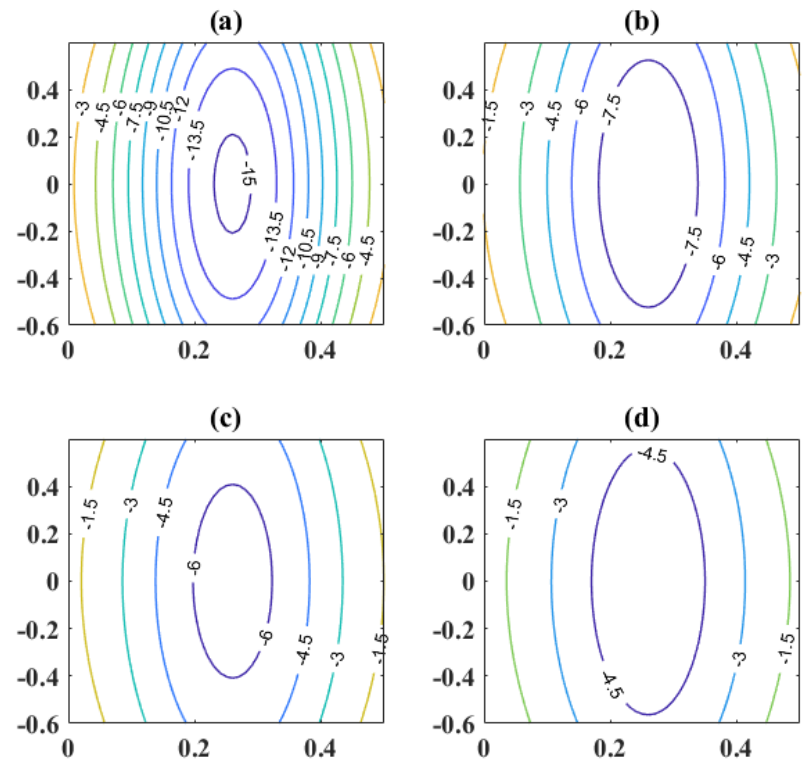

Figure 9. Streamlines for varying (a) $\alpha=0.1$, (b) $\alpha=0.2$, (c) $\alpha=0.3$ and (d) $\alpha=0.4$.

The effects of $\lambda_{1}, \varepsilon, D a, \phi, B i$ and $B r$ on temperature are plotted in Fig. 5. The temperature profiles are non-parabolic and show the dual nature. The role of $\lambda_{1}$ on temperature is shown in Fig. 5(a). The temperature decreases with increasing values of $\lambda_{1}$. Finally, the behavior of $\varepsilon$ on temperature 
G. Manjunatha et al.

International Journal of Thermofluid Science and Technology (2020), Volome 7, Issue 1, Paper No. 20070101

shows the opposite trend as that of $\lambda_{1}$ (Fig. 5(b)). The role of $D a$ on temperature is examined in Fig. 5(c). It is observed that the increasing values of $D a$ decrease the temperature near the axis of the tube and opposite behavior is observed near the walls. The effect of $\phi$ plays a vital role in the determination of temperature. It means that a larger value of $\phi$ increases temperature near the axis of the tube and the effect is negligible near the walls (See Fig. 5(d)). This is because of an increase in the value of $\phi$ allows the fluid to dissipate or absorb heat to its surroundings. Hence, an increase in $\phi$ decreases the temperature near the walls of the tube. Fig. 5(e) depicts the variation of temperature due to the influence of $B i$. An increase in the value of $B i$ results in the reduction of temperature. Fig. 5(f) illustrates the effect of $B r$ on temperature. It is found that an increase in the value of $\mathrm{Br}$ increases temperature. Since $E c$ occurs due to the viscous dissipation effects and it enhances temperature. Further, an increase in the value of Pr decrease thermal conductivity and thereby increases temperature.

The essential part of peristalsis is trapping. It is by and large the arrangement of the inside flowing bolus. These trapped boluses push ahead alongside the sinusoidal movement of the peristaltic wave. This phenomenon is particularly useful in bloodstream issues, for example, the arrangement of thrombus and transport of food bolus in the gastrointestinal tract. Figs. 6-8 shows that the size of trapped bolus decreases as we increase the value of $\lambda_{1}, D a$ and $\varepsilon$ and hence it increases the number of bolus formations. Whereas it increases with an increase in the value of $\alpha$ and thereby, it vanishes the trapped bolus for larger values of $\alpha$ which is observed in Fig. 9. From these figures, one can observe that the bolus moves at the same speed as that of the wave.

\section{Conclusion}

It is worth noticing that, from the current model, one can deduce the results of a Newtonian model by taking $\lambda_{1}=0$. The present study provides a satisfactory outcome that represents some of the natural phenomena, especially the flow of blood in narrow arteries, which can be handled and processed in case of dysfunction. The conclusions can be summarized as follows:

* The axial velocity increases with an expansion in the estimation of the porous parameter, and it diminishes for a larger value of velocity slip parameter.

* The flow rate in an incline elastic tube increases with an expansion in the porous parameter, and it diminishes with an increment in the slip parameter.

* The influence of the Jeffery parameter and angle of inclination enhances the flow rate.

- The effects of elastic parameters, outlet elastic radius and amplitude ratio increases the flow rate while the inlet elastic parameter decreases the flow rate.

* The magnitude of temperature diminishes with an expansion in the Biot number and the temperature depended thermal conductivity increases the temperature close to the axis and the impact is irrelevant close to the walls.

* The volume of tapered bolus diminishes with expanding porous parameter, and it increments for larger values of the velocity slip parameter.

Acknowledgments: The authors appreciate the constructive comments of the reviewers which led to definite improvement in the paper. 


\section{References}

Abbsi, F. M., Hayat, T. \& Ahmad, B. (2015). Numerical analysis for peristaltic transport of CarreauYasuda fluid with variable thermal conductivity and convective conditions. Journal of Central South University of Technology, 22, 4467-4475.

Abdul MalequeKh. (2017). Temperature Dependent Suction/Injection and Variable Properties on Non-Newtonian Casson Mixed Convective MHD Laminar Fluid Flow with Viscous Dissipation and Thermal Radiation. American Journal of Heat and Mass Transfer, 4 (2), 104120.

Alsaedi, A., Batool, N., Yasmin, H. \&Hayat, T. (2013). Convective heat transfer analysis on Prandtl fluid model with peristalsis. Applied Bionics and Biomechanics, 10,197-208.

Bhatti, M. M. \&Abbas, M. A. (2016). Simultaneous effects of slip and MHD on peristaltic blood flow of Jeffrey fluid model through a porous medium. AlexandriaEngineering Journal, 55, 10171023.

Burns, J. C. \& Parkes, T. (1967). Peristaltic motion. Journal of Fluid Mechanics, 29, 731-743.

El-Koumy, S. R., Barakat, E. S. I. \&Abdelsalam, S. I. (2012). Hall and porous boundaries effects on peristaltic transport through porous medium of a Maxwell model. Transport in Porous Media, 94, 643-658.

El-Shehawey, E. F., Mekheimer, K., Kaldas, S. \&Afifi, N. (1999). Peristaltic transport through a porous medium. Journal of Biomathematics, 14.

Hayat, T., Ali, N. \& Asghar, S. (2007). Ananalysis of peristaltic transport for flow of a Jeffery fluid. Acta Mechanica, 193, 101-112.

Hayat, T., Farooq, S., Ahmad, B. \&Alsaedi, A. (2016). Characteristics of convective heat transfer in the MHD peristalsis of Carreau fluid with Joule heating. AIP Advances, 6, 045302.

Hayat, T., Yasmin, H., Alhuthali, M. \&Kutbi, M. (2013). Peristaltic Flow of a Non-Newtonian Fluid in an Asymmetric Channel with Convective Boundary Conditions. Journal of Mechanics, 29, 599-607.

Jaggy, C., Lachat, M, Leskosek, B., Znd, G., \&Turina, M. (2000). Affinity pump system: a new peristaltic blood pump for cardiopulmonary bypass. Perfusion, 15, 77-83.

Latham, T. W. (1966). Fluid motion in peristaltic pump, MS, thesis. Massachusetts Institute of Technology.

Manjunatha, G. \& Rajashekhar, C. (2018). Slip effects on peristaltic transport of Casson fluid in an inclined elastic tube with porous walls. Journal of Advanced Fluid Mechanics and Thermal Sciences, 43, 67-80.

Manjunatha, G., Rajashekhar, C., Vaidya, H. \& Prasad, K.V. (2019) (a). Peristaltic mechanism of Bingham liquid in A convectively heated porous tube in the Presence of variable liquid properties. Special Topics \& Reviews in Porous Media: An International Journal, 10, 187-201.

Manjunatha, G., Rajashekhar, C., Vaidya, H., Prasad, K. V., Makinde, O. D. \&Viharika, J. U. (2019) (b). Impact of variable transport properties and slip effects on MHD Jeffrey fluid through channel. Arabian Journal for Science and Engineering.

Manjunatha, G., Rajashekhar, C., Vaidya, H., Prasad, K.V. \& Vajravelu, K. (2019) (c). Impact of heat and mass transfer on the peristaltic mechanism of Jeffrey fluid in a non-uniform porous 
G. Manjunatha et al.

International Journal of Thermofluid Science and Technology (2020), Volome 7, Issue 1, Paper No. 20070101

channel with variable viscosity and variable thermal conductivity. Journal of Thermal Analysis and Calorimetry.

Nadeem, S. \&Akram, S. (2010). Peristaltic flow of a Jeffrey fluid in a rectangular duct. Nonlinear Analysis: Real World Applications, 11, 4238-4247.

Nadeem, S. \&Akram, S. (2011). Peristaltic flow of a Maxwell model through porous boundaries in a porous medium. Transport in Porous Media, 86, 895-900.

Prasad, K. V., Vajravelu, K., Hanumesh Vaidya, Rashidi, M. M. \&Neelufer.Z. Basha. (2018) (a). Flow and Heat Transfer of a Casson Liquid over a Vertical Stretching Surface: Optimal Solution. American Journal of Heat and Mass Transfer, 5 (1), 1-22.

Prasad, K. V., Vajravelu, K., Vaidya, H., Basha, N. Z. \& Umesh, V.(2017). Thermal and species concentration of MHD Casson fluid at a vertical sheet in the presence variable fluid properties. Ain Shams Engineering Journal.

Prasad, K. V., Vaidya, H., Vajravelu, K.\& Ramanajini, V. R. (2018) (b). Analytical study of CattaneoChristov Heat Flux Model for Williamson-Nanofluid Flow Over a Slender Elastic Sheet with Variable Thickness. Journal of Nanofluids, 7, 583-594.

Raju, K. K. \&Devanathan, R. (1972). Peristaltic motion of a non-Newtonian fluid.Rheologica Acta, $11,170-178$.

Rubinow, S. I. \& Keller, J. B. (1972). Flow of a viscous fluid through an elastic tube with application to blood flow. Journal of Theoretical Biology,35, 299-313.

Saffman, P. G. (1971). On the Boundary conditions at the surface of a porous medium. Studies in Applied Mathematics,1, 93-101.

Sayed, H. M., Aly, E. H. \& Vajravelu, K. (2016). Influence of slip and convective boundary conditions on peristaltic transport of non-Newtonian nanofluids in an inclined asymmetric channel. Alexandria Engineering Journal, 55, 2209-2220.

Selvi, C. K., Haseena, C., Srinivas, A. N. S. \& Sreenadh, S. (2017). The effect of heat transfer on peristaltic flow of Jeffrey fluid in an inclined porous stratum. IOP Conf. Series: Materials Science and Engineering, 263, 062027.

Srinivasa Raju R. (2017). Application of Finite Element Method to MHD Mixed Convection Chemically Reacting Flow past a Vertical Porous Plate with Cross Diffusion and Biot Number Effects. $\quad$ American Journal of Heat and Mass Transfer, 4 (3), 53-74.

Tripathi, D. \& Beg, O. A. (2012). A numerical study of oscillating peristaltic flow of generalized Maxwell viscoelastic fluids through porous medium. Transport in Porous Media, 95, 337348.

Vaidya, H., Choudhari, R., Gudekote, M. \& Prasad, K.V. (2019) (a). Effect of variable liquid properties on peristaltic transport of Rabinowitsch liquid in convectively heated complaint porous channel. Journal of Central South University, 26, 1116-1132.

Vaidya, H., Rajashekhar, C., Manjunatha, G. \& Prasad, K.V. (2019) (b). Effect of variable liquid properties on peristaltic flow of a Rabinowitsch fluid in an inclined convective porous channel. The European Physical Journal Plus, 134, 231.

Vaidya, H., Rajashekhar, C., Manjunatha, G. \& Prasad, K.V. (2019) (c). Peristaltic mechanism of a Rabinowitsch fluid in an inclined channel with complaint wall and variable liquid properties. Journal of the Brazilian Society of Mechanical Sciences and Engineering, 41, 52.

Vaidya, H., Rajashekhar, C., Manjunatha, G., Prasad, K.V., Makinde, O.D. \&Sreenadh, S. (2019) (d). Peristaltic motion of non-Newtonian fluid with variable liquid properties in a convectively 
G. Manjunatha et al.

International Journal of Thermofluid Science and Technology (2020), Volome 7, Issue 1, Paper No. 20070101

heated non-uniform tube: Rabinowitsch fluid model. Journal of Enhanced Heat Transfer, 26, 277-294.

Vajravelu, K., Prasad, K. V., Vaidya, H., Basha, N.Z. \& Ng, Chiu-On. (2016) (a). Mixed Convective Flow of a Casson Fluid over a Vertical Stretching Sheet. InternationalJournal of Applied and Computational Mathematics, 3, 1619-1638.

Vajravelu, K., Sreenadh, S. \&Lakshminarayana, P. (2011). The influence of heat transfer on peristaltic transport of a Jeffrey fluid in a vertical porous stratum. Communications in Non-linear Science and Numerical Simulation, 16 (8), 3107-3125.

Vajravelu, K., Sreenadh, S., Lakshminarayana, P., Sucharitha, G. \&Rashidi, M. M. (2016) (b). Peristaltic Flow of Phan-Thien-Tanner Fluid in an Asymmetric Channel with Porous Medium. Journal of Applied Fluid Mechanics, 9, 1615-1625.

Young, DF. (1968). Effects of a time-dependent stenosis on flow through a tube. J. Engg. Ind. Trans. ASME., 90, 248-254. 